PROCEEDINGS OF THE

AMERICAN MATHEMATICAL SOCIETY

Volume 128, Number 2, Pages 589-598

S 0002-9939(99)05332-0

Article electronically published on July 23, 1999

\title{
CHARACTERIZATIONS OF RIEMANNIAN SPACE FORMS, EINSTEIN SPACES AND CONFORMALLY FLAT SPACES
}

\author{
BANG-YEN CHEN, FRANKI DILLEN, LEOPOLD VERSTRAELEN, AND \\ LUC VRANCKEN
}

(Communicated by Christopher Croke)

\begin{abstract}
In a recent paper the first author introduced two sequences of Riemannian invariants on a Riemannian manifold $M$, denoted respectively by $\delta\left(n_{1}, \ldots, n_{k}\right)$ and $\hat{\delta}\left(n_{1}, \ldots, n_{k}\right)$, which trivially satisfy $\delta\left(n_{1}, \ldots, n_{k}\right) \geq$ $\hat{\delta}\left(n_{1}, \ldots, n_{k}\right)$. In this article, we completely determine the Riemannian manifolds satisfying the condition $\delta\left(n_{1}, \ldots, n_{k}\right)=\hat{\delta}\left(n_{1}, \ldots, n_{k}\right)$. By applying the notions of these $\delta$-invariants, we establish new characterizations of Einstein and conformally flat spaces; thus generalizing two well-known results of Singer-Thorpe and of Kulkarni.
\end{abstract}

\section{INTRODUCTION}

Riemannian invariants are the intrinsic characteristics of the Riemannian manifold. Among all Riemannian invariants, curvature is "the $\mathrm{N}^{o} 1$ Riemannian invariant and the most natural", according to Marcel Berger in [1].

Classically, among the Riemannian curvature invariants, people have been studying sectional, scalar and Ricci curvatures. In [3], the first author introduces new types of curvature invariants, defining two strings of scalar-valued Riemannian curvature functions $M \rightarrow \mathbb{R}$, namely $\delta\left(n_{1}, \ldots, n_{k}\right)$ and $\hat{\delta}\left(n_{1}, \ldots, n_{k}\right)$ for every $\left(n_{1}, \ldots, n_{k}\right)$ satisfying $n_{1}<n, n_{j} \geq 2$ and $n_{1}+\cdots+n_{k} \leq n$. For these two strings of Riemannian invariants, one always has trivially (see below)

$$
\delta\left(n_{1}, \ldots, n_{k}\right) \geq \hat{\delta}\left(n_{1}, \ldots, n_{k}\right) .
$$

When a Riemannian manifold $M$ is immersed as a submanifold in some larger Riemannian manifold $\widetilde{M}$, the shape that the submanifold $M$ assumes in $\widetilde{M}$ is determined by various extrinsic curvatures. Among the scalar-valued extrinsic curvature functions $M \rightarrow \mathbb{R}$, the squared mean curvature $H^{2}$ has so far been studied most intensively, due to its natural relation to geometric variational problems on area and volume and to the physical notion of tension. In particular, all sorts of shapes assumed by surfaces in the classical Euclidean world $\mathbb{E}^{3}$, from soap bubbles to red blood cells, seem to be determined by various curvatures. For a beautiful survey on extrinsic and intrinsic curvatures, we refer to the article [6] of Robert Osserman.

Received by the editors April 17, 1997.

1991 Mathematics Subject Classification. Primary 53B20; Secondary 53C42.

Key words and phrases. Curvature, conformally flat space, Einstein space, $\delta$-invariants.

The second and fourth authors were supported by a postdoctoral Fellow of the Fund for Scientific Research - Flanders (Belgium)(F.W.O.). Research supported by OT/TBA/95/9.

(c)1999 American Mathematical Society 
It was shown by the first author in [3] that, for any isometric immersion of a Riemannian $n$-manifold $M$ in a Riemannian space form $R^{m}(\epsilon)$ of constant sectional curvature $\epsilon, \delta\left(n_{1}, \ldots, n_{k}\right)$ satisfies the following sharp inequality:

$$
\delta\left(n_{1}, \ldots, n_{k}\right) \leq \frac{n^{2}\left(n+k-1-\sum n_{j}\right)}{2\left(n+k-\sum n_{j}\right)} H^{2}+\frac{1}{2}\left(n(n-1)-\sum_{j=1}^{k} n_{j}\left(n_{j}-1\right)\right) \epsilon .
$$

Combining (1.1) and (1.2) one also has the following inequality:

$$
\hat{\delta}\left(n_{1}, \ldots, n_{k}\right) \leq \frac{n^{2}\left(n+k-1-\sum n_{j}\right)}{2\left(n+k-\sum n_{j}\right)} H^{2}+\frac{1}{2}\left(n(n-1)-\sum_{j=1}^{k} n_{j}\left(n_{j}-1\right)\right) \epsilon .
$$

According to the imbedding theorem of John Nash, every given Riemannian manifold $M$ can be realized as a submanifold in sufficiently large Euclidean ambient space. Inequalities (1.2), (1.3) then give "for free" (the inequalities hold without imposing any condition at all) optimal relations between some of the main scalarvalued intrinsic and extrinsic curvature invariants. These inequalities among others give answers to some old and basic questions (like which Riemannian characteristics of $M$ obstruct to minimal immersibility in some Euclidean space), give new views on rigidity of submanifolds as expressed in the new notion of "ideal immersions", and give new results on purely intrinsic spectral properties of homogeneous spaces obtained via extrinsic data. For these and other observations concerning the significant role played by the strings of invariants, we refer to [2], [3], [4].

In this article, we classify Riemannian manifolds which satisfy the equality case of (1.1), i.e., satisfy the condition: $\delta\left(n_{1}, \ldots, n_{k}\right)=\hat{\delta}\left(n_{1}, \ldots, n_{k}\right)$. Further we apply the notion of the scalar curvature of $k$-plane sections to establish a simple characterization of conformally flat spaces, which generalizes a well-known result of R. S. Kulkarni [5]. We also obtain a characterization of the even-dimensional Einstein manifolds in terms of scalar curvatures of $r$-plane sections, which extends a well-known characterization of Einstein 4-manifolds due to I. M. Singer and J. A. Thorpe [7]. Finally we determine all submanifolds of a Riemannian space form $R^{m}(\epsilon)$ which realize the equality in (1.3).

\section{RIEMANNIAN INVARIANTS}

Let $M$ be a Riemannian $n$-manifold. Denote by $K(\pi)$ the sectional curvature of $M$ associated with a plane section $\pi \subset T_{p} M, p \in M$. For any orthonormal basis $e_{1}, \ldots, e_{n}$ of the tangent space $T_{p} M$, the scalar curvature $\tau$ at $p$ is in a perhaps somewhat non-standardly normalized way, which is rather suitable for our purposes, defined to be

$$
\tau(p)=\sum_{i<j} K\left(e_{i} \wedge e_{j}\right)
$$

If $L$ is a subspace of dimension $r \geq 2$ in $T_{p} M$, the scalar curvature $\tau(L)$ of $L$ is defined in [3] to be

$$
\tau(L)=\sum_{\alpha<\beta} K\left(e_{\alpha} \wedge e_{\beta}\right), \quad 1 \leq \alpha, \beta \leq r
$$


where $\left\{e_{1}, \ldots, e_{r}\right\}$ is an orthonormal basis of $L$. The scalar curvature $\tau(p)$ of $M$ at $p$ is nothing but the scalar curvature of the tangent space of $M$ at $p$; and if $L$ is a 2-plane, $\tau(L)$ is the sectional curvature $K(L)$ of $L$. In general, $\tau(L)$ is the scalar curvature of the image $\exp _{p}(L)$ of $L$ at $p$ under the exponential map at $p$. The notion of scalar curvature of a general $r$-plane section plays a central role in this article.

For an integer $k \geq 0$, denote by $\mathcal{S}(n, k)$ the set consisting of all $k$-tuples $\left(n_{1}, \ldots, n_{k}\right)$ of integers $\geq 2$ satisfying $n_{1}<n$ and $n_{1}+\cdots+n_{k} \leq n$. Let $\mathcal{S}(n)$ denote the union of all $\mathcal{S}(n, k), k \geq 0$. For each $k$-tuple $\left(n_{1}, \ldots, n_{k}\right) \in \mathcal{S}(n, k)$, Riemannian invariants $S_{\left(n_{1}, \ldots, n_{k}\right)}(p)$ and $\hat{S}_{\left(n_{1}, \ldots, n_{k}\right)}(p)$ are defined respectively by

$$
\begin{aligned}
& S_{\left(n_{1}, \ldots, n_{k}\right)}(p)=\inf \left\{\tau\left(L_{1}\right)+\cdots+\tau\left(L_{k}\right)\right\}, \\
& \hat{S}_{\left(n_{1}, \ldots, n_{k}\right)}(p)=\sup \left\{\tau\left(L_{1}\right)+\cdots+\tau\left(L_{k}\right)\right\},
\end{aligned}
$$

where $L_{1}, \ldots, L_{k}$ run over all $k$ mutually orthogonal subspaces of $T_{p} M$ such that $\operatorname{dim} L_{j}=n_{j}, j=1, \ldots, k$.

The Riemannian invariants $\delta\left(n_{1}, \ldots, n_{k}\right)(p)$ and $\hat{\delta}\left(n_{1}, \ldots, n_{k}\right)(p)$ introduced in [3] are given by

$$
\begin{aligned}
& \delta\left(n_{1}, \ldots, n_{k}\right)(p)=\tau(p)-S_{\left(n_{1}, \ldots, n_{k}\right)}(p), \\
& \hat{\delta}\left(n_{1}, \ldots, n_{k}\right)(p)=\tau(p)-\hat{S}_{\left(n_{1}, \ldots, n_{k}\right)}(p) .
\end{aligned}
$$

Clearly, $\delta\left(n_{1}, \ldots, n_{k}\right) \geq \hat{\delta}\left(n_{1}, \ldots, n_{k}\right)$ for any $k$-tuple $\left(n_{1}, n_{2}, \ldots, n_{k}\right) \in \mathcal{S}(n, k)$. For simplicity, a Riemannian manifold $M$ is called an $S\left(n_{1}, \ldots, n_{k}\right)$-space if it satisfies $\delta\left(n_{1}, \ldots, n_{k}\right)=\hat{\delta}\left(n_{1}, \ldots, n_{k}\right)$ identically. It follows from (2.3) and (2.4) that a Riemannian $n$-manifold is an $S\left(n_{1}, \ldots, n_{k}\right)$-space if and only if $\tau\left(L_{1}\right)+\cdots+\tau\left(L_{k}\right)$ is independent of the choice of $k$ mutually orthogonal subspaces $L_{1}, \ldots, L_{k}$ which satisfy $\operatorname{dim} L_{j}=n_{j}, j=1, \ldots, k$.

Finally, we introduce some notation. Let $\left\{e_{1}, \ldots, e_{n}\right\}$ be any orthonormal basis of $T_{p} M$. Then we denote the scalar curvature of the $j$-space spanned by $e_{i_{1}}, \ldots, e_{i_{j}}$ by $\tau_{i_{1} \cdots i_{j}}$.

\section{Generalizations of Kulkarni's Characterization of COnformally FLAT SPACES AND Singer-ThORPE'S CHARACTERIZATION OF EINSTEIN SPACES}

We first prove the following generalization of a result of Kulkarni [5].

Theorem 1. Let $M^{n}$ be a Riemannian manifold with $n \geq 4$, and let $s$ be any integer satisfying $2<2 s \leq n$. Then $M$ is conformally flat if and only if for any orthonormal set $\left\{e_{1}, \ldots, e_{2 s}\right\}$ of vectors one has

$$
\tau_{1 \cdots s}+\tau_{s+1 \cdots 2 s}=\tau_{1 \cdots s-1 s+1}+\tau_{s s+2 \cdots 2 s} .
$$

Proof. For $s=2$ this is Kulkarni's result. For completeness, we include a proof of this case.

If $M$ is conformally flat, Weyl's conformal curvature tensor vanishes. Thus

$$
K_{i j}=\frac{1}{n-2}\left(\operatorname{Ric}\left(e_{i}\right)+\operatorname{Ric}\left(e_{j}\right)\right)-\frac{\tau}{(n-1)(n-2)}, \quad i \neq j,
$$

from which we conclude that

$$
K_{i j}+K_{k \ell}=K_{i k}+K_{j \ell}, \quad \text { for distinct } i, j, k, \ell \text {. }
$$


Conversely, if (3.3) holds for distinct $i, j, k, \ell$, then, by fixing $i, j, k$ in (3.3) and summing up over all remaining $\ell$, one obtains

$$
(n-2) K_{i j}+\operatorname{Ric}\left(e_{k}\right)=(n-2) K_{i k}+\operatorname{Ric}\left(e_{j}\right) .
$$

Fixing $i, j$ in (3.4) and summing up over all remaining $k$, we obtain (3.2), which implies the vanishing of Weyl's conformal curvature tensor. Therefore, $M$ is conformally flat.

Now, we prove the theorem for $s>2$. First we prove that a conformally flat space satisfies (3.1). So suppose that $M^{n}$ is conformally flat and let $s$ be any integer satisfying $2<2 s \leq n$.

From Kulkarni's result we know that $K_{i s}+K_{k s+1}=K_{i s+1}+K_{k s}$ for any $i<s$ and $k>s+1$. Therefore we have that

$$
\begin{gathered}
\tau_{1 \cdots s}+\tau_{s+1 \cdots 2 s}=\tau_{1 \cdots s-1}+\sum_{i=1}^{s-1}\left(K_{i s}+K_{s+1} s+1+i\right)+\tau_{s+2 \cdots 2 s} \\
=\tau_{1 \cdots s-1}+\sum_{i=1}^{s-1}\left(K_{i s+1}+K_{s s+1+i}\right)+\tau_{s+2 \cdots 2 s} \\
=\tau_{1 \cdots s-1 s+1}+\tau_{s j+2 \cdots 2 s} .
\end{gathered}
$$

Next we prove (3.1) implies conformal flatness. For this we use (3.1) twice to obtain

$$
\begin{aligned}
0= & \left(\tau_{1 \cdots s}+\tau_{s+1 \cdots 2 s}\right)-\left(\tau_{1 \cdots s-1 s+1}+\tau_{s s+2 \cdots 2 s}\right) \\
& -\left(\left(\tau_{1 \cdots s-2 s+2 s}+\tau_{s+1 s-1 s+3 \cdots 2 s}\right)\right. \\
& \left.-\left(\tau_{1 \cdots s-2 s+2 s+1}+\tau_{s s-1 s+3 \cdots 2 s}\right)\right) .
\end{aligned}
$$

It is clear that $K_{i k}$ does not occur in (3.5) unless both $i$ and $k$ belong to the set $\{s-1, s, s+1, s+2\}$. Taking this into consideration, (3.5) becomes

$$
0=2\left(\left(K_{s-1 s}+K_{s+1 s+2}\right)-\left(K_{s-1 s+1}+K_{s s+2}\right)\right),
$$

and Kulkarni's result implies that $M$ is conformally flat.

In particular, statement (3.1) yields the following.

Corollary 1. Let $M$ be a Riemannian 2r-manifold with $r>1$. Then $M$ is a conformally flat manifold with vanishing scalar curvature if and only if $\tau(L)=$ $-\tau\left(L^{\perp}\right)$ for any $r$-plane section $L \subset T_{p} M, p \in M$, where $L^{\perp}$ denotes the orthogonal complement of $L$ in $T_{p} M$.

We remark that a manifold is conformally flat with vanishing scalar curvature if and only if it is conharmonically flat. Hence Corollary 1 is a generalization of a result in [8].

The following result generalizes a well-known characterization of 4-dimensional Einstein spaces due to I. M. Singer and J. A. Thorpe [7].

Theorem 2. Let $M$ be a Riemannian $2 \ell$-manifold. Then $M$ is an Einstein space if and only if $\tau(L)=\tau\left(L^{\perp}\right)$ for any $\ell$-plane section $L \subset T_{p} M$, where $L^{\perp}$ denotes the orthogonal complement of $L$ in $T_{p} M$.

Proof. Let $L$ be an arbitrary $\ell$-plane section at $p$. Choose an orthonormal basis $\left\{e_{1}, \ldots, e_{2 \ell}\right\}$ at $p$ such that $L$ is spanned by $e_{1}, \ldots, e_{\ell}$.

If $M$ is an Einstein space, then the Ricci curvatures of $M$ satisfy

$$
\operatorname{Ric}\left(e_{1}\right)+\cdots+\operatorname{Ric}\left(e_{\ell}\right)=\operatorname{Ric}\left(e_{\ell+1}\right)+\cdots+\operatorname{Ric}\left(e_{2 \ell}\right) .
$$


Equation (3.6) yields $\tau(L)=\tau\left(L^{\perp}\right)$.

Conversely, suppose that we have $\tau(L)=\tau\left(L^{\perp}\right)$ for any $\ell$-plane section $L \subset$ $T_{p} M$. Then we have

$$
\tau_{12 \cdots \ell}-\tau_{2 \cdots \ell+1}=\tau_{\ell+1 \cdots 2 \ell}-\tau_{1 \ell+2 \cdots 2 \ell} .
$$

Equation (3.7) implies

$$
\begin{aligned}
K_{12} & +\cdots+K_{1 \ell}-\left(K_{(\ell+1) 2}+\cdots+K_{(\ell+1) \ell}\right) \\
& =K_{(\ell+1)(\ell+2)}+\cdots+K_{(\ell+1)(2 \ell)}-\left(K_{1(\ell+2)}+\cdots+K_{1(2 \ell)}\right),
\end{aligned}
$$

which yields $\operatorname{Ric}\left(e_{1}\right)=\operatorname{Ric}\left(e_{\ell+1}\right)$. Since $\ell>1$, this implies that $M$ is Einstein.

\section{Riemannian space forms, Einstein spaces AND CONFORMALLY FLAT SPACES}

In this section, we characterize Riemannian space forms, Einstein spaces and conformally flat spaces as being $S$-spaces. In fact we classify all $S\left(n_{1}, \ldots, n_{k}\right)$ spaces. Clearly if $k=0$, there is nothing to show. Hence if the dimension of the manifold is 2 , there is also nothing to show.

First we consider $S(j)$-spaces. We start with some lemmas.

Lemma 1. For a given integer $j$ with $2 \leq j \leq n-2$, if $M$ is an $S(j)$-space, then it is an $S(j+1)$-space.

Proof. For simplicity, we start with a special case: $j=3$. If $M$ is an $S(3)$-space, then $\tau_{123}$ is a number, say $c$, which is independent of the choice of the 3-plane. In particular, from the definition of scalar curvature of a $j$-plane, we have

$$
\tau_{234}=\tau_{1234}-K_{12}-K_{13}-K_{14}=c,
$$

where $K_{i j}$ denotes the sectional curvature of the 2-plane spanned by $e_{i}, e_{j}$.

On the other hand, since $M$ is an $S(3)$-space, we have

$$
\begin{aligned}
& K_{12}+K_{13}+K_{23}=c, \\
& K_{13}+K_{14}+K_{34}=c, \\
& K_{12}+K_{14}+K_{24}=c .
\end{aligned}
$$

Summing up (4.2)-(4.4) we obtain

$$
\tau_{1234}+K_{12}+K_{13}+K_{14}=3 c .
$$

Combining (4.1) and (4.5) yields $\tau_{1234}=2 c$. Since the orthonormal basis can be chosen arbitrarily, this implies that $M$ is an $S(4)$-space.

In general, if $M$ is an $S(j)$-space, then

$$
\tau_{2 \cdots j+1}=\tau_{1 \cdots j+1}-K_{12}-\cdots-K_{1 j+1}=c .
$$

On the other hand, similar to (4.5), we also have

$$
(j-2) \tau_{1 \cdots j+1}+K_{12}+\cdots+K_{1 j+1}=j c .
$$

Combining (4.6) and (4.7) yields $(j-1) \tau_{1 \cdots j+1}=(j+1) c$. This implies $M$ is an $S(j+1)$-space.

Lemma 2. $M$ is an $S(n-1)$-space if and only if $M$ is an Einstein space. 
Proof. This Lemma follows obviously from the following identity:

$$
\tau\left(L^{n-1}\right)=\tau-\operatorname{Ric}\left(e_{n}\right),
$$

where $e_{n}$ is a unit vector perpendicular to a hyperplane $L^{n-1} \subset T_{p} M, p \in M$.

Lemma 3. An $S(n-2)$-space is a Riemannian space form.

Proof. Let $M$ be an $S(n-2)$-space. Then $M$ is an Einstein space according to Lemmas 1 and 2 . On the other hand, let $e_{n-1}, e_{n}$ be any orthonormal vectors at a point $p$ and let $L^{n-2}$ be the $(n-2)$-subspace of the tangent space $T_{p} M$ perpendicular to $e_{n-1}$ and $e_{n}$. We have

$$
\tau-\operatorname{Ric}\left(e_{n-1}\right)-\operatorname{Ric}\left(e_{n}\right)+K_{n-1 n}=c,
$$

where $c$ is independent of the choice of $L^{n-2}$. Because $M$ is Einstein, (4.8) implies that $K_{n-1 n}$ does not depend on the choice of the plane $e_{n-1} \wedge e_{n}$. Hence $M$ is a Riemannian space form. This completes the proof of Lemma 3.

Thus we have proved the following theorem.

Theorem 3. Let $M$ be a Riemannian n-manifold with $n>2$. Then

1. For any integer $j$ with $2 \leq j \leq n-2, M$ is an $S(j)$-space if and only if $M$ is a Riemannian space form.

2. $M$ is an $S(n-1)$-space if and only if $M$ is an Einstein space.

Now we consider $S\left(n_{1}, \ldots, n_{k}\right)$-spaces for $k>1$. We will prove the following theorem.

Theorem 4. Let $M$ be a Riemannian n-manifold and $k$ an integer $\geq 2$.

1. If $M$ is an $S\left(n_{1}, \ldots, n_{k}\right)$-space, then $M$ is a Riemannian space form unless $n_{1}=\ldots=n_{k}$ and $n_{1}+\cdots+n_{k}=n$.

2. $M$ is an $S\left(n_{1}, \ldots, n_{k}\right)$-space with $n_{1}=\ldots=n_{k}$ and $n_{1}+\cdots+n_{k}=n$ if and only if $M$ is a conformally flat space.

Proof. Without loss of generality, we may assume $n_{1} \leq n_{2} \leq \cdots \leq n_{k}$. Let $e_{1}, \ldots, e_{n}$ be an orthonormal basis of $T_{p} M$. If $M$ is an $S\left(n_{1}, \ldots, n_{k}\right)$-space, then we have

$$
\tau_{12 \cdots n_{1}}+\cdots+\tau_{n_{1}+\cdots+n_{k-1}+1 \cdots n_{1}+\cdots+n_{k}}=c,
$$

where $c$ is independent of the orthonormal basis.

Consider a permutation $\sigma$ of $\left\{1, \ldots, n_{1}+\cdots+n_{k}\right\}$. For each such permutation, there exists a corresponding equation similar to (4.9) given by

$$
\tau_{\sigma(1) \sigma(2) \cdots \sigma\left(n_{1}\right)}+\cdots+\tau_{\sigma\left(n_{1}+\cdots+n_{k-1}+1\right) \cdots \sigma\left(n_{1}+\cdots+n_{k}\right)}=c .
$$

By summing up all such equations we obtain

$$
c_{1} \tau_{12 \cdots n_{1}+\cdots+n_{k}}=c_{2} c,
$$

where $c_{1}, c_{2}$ are some positive constants. This implies that $M$ is an $S\left(n_{1}+\cdots+n_{k}\right)$ space. Thus, according to Theorem 3 , if $n_{1}+\cdots+n_{k} \leq n-2$, then $M$ is a Riemannian space form; if $n_{1}+\cdots+n_{k}=n-1$, then $M$ is an Einstein space; if $n_{1}+\cdots+n_{k}=n$, we obtain no new information. In the first case, we are done, so we only have to consider the last two cases. First we will assume that $M$ is not Einstein, and prove that $M$ has to be conformally flat. Next we will show that if $M$ is Einstein, then $M$ has constant sectional curvature. 
Case 1. $M$ is not Einstein. Clearly, in this case, we must have $n_{1}+\cdots+n_{k}=$ $n$, so (4.9) becomes

$$
\tau_{12 \cdots n_{1}}+\cdots+\tau_{n_{1}+\cdots+n_{k-1}+1 \cdots n}=c .
$$

Consider a permutation $\sigma$ of $\{2, \ldots, n\}$. For each such permutation, there is a corresponding equation similar to (4.12) given by

$$
\tau_{1 \sigma(2) \cdots \sigma\left(n_{1}\right)}+\cdots+\tau_{\sigma\left(n_{1}+\cdots+n_{k-1}+1\right) \cdots \sigma(n)}=c .
$$

By summing up all such equations we obtain

$$
c_{3} \operatorname{Ric}\left(e_{1}\right)+c_{4} \tau_{2, \ldots, n}=c_{5} c
$$

where

$$
\begin{aligned}
c_{3} & =\left(\begin{array}{c}
n-2 \\
n_{1}-2
\end{array}\right)\left(\begin{array}{c}
n-n_{1} \\
n_{2}
\end{array}\right) \cdots\left(\begin{array}{c}
n-n_{1}-\cdots-n_{k-1} \\
n_{k}
\end{array}\right), \\
c_{4} & =\left(\begin{array}{c}
n-3 \\
n_{1}-3
\end{array}\right)\left(\begin{array}{c}
n-n_{1} \\
n_{2}
\end{array}\right) \cdots\left(\begin{array}{c}
n-n_{1}-\cdots-n_{k-1} \\
n_{k}
\end{array}\right) \\
& +\left(\begin{array}{c}
n-3 \\
n_{2}-2
\end{array}\right)\left(\begin{array}{c}
n-n_{2}-1 \\
n_{1}-1
\end{array}\right)\left(\begin{array}{c}
n-n_{1}-n_{2} \\
n_{3}
\end{array}\right) \cdots\left(\begin{array}{c}
n-n_{1}-\cdots-n_{k-1} \\
n_{k}
\end{array}\right) \\
& +\cdots \\
& +\left(\begin{array}{c}
n-3 \\
n_{k}-2
\end{array}\right)\left(\begin{array}{c}
n-n_{k}-1 \\
n_{1}-1
\end{array}\right)\left(\begin{array}{c}
n-n_{1}-n_{k} \\
n_{2}
\end{array}\right) \cdots\left(\begin{array}{c}
n-n_{1}-\cdots-n_{k-2}-n_{k} \\
n_{k-1}
\end{array}\right), \\
c_{5} & =\left(\begin{array}{c}
n-1 \\
n_{1}-1
\end{array}\right)\left(\begin{array}{c}
n-n_{1} \\
n_{2}
\end{array}\right) \cdots\left(\begin{array}{c}
n-n_{1}-\cdots-n_{k-1} \\
n_{k}
\end{array}\right),
\end{aligned}
$$

where $\left(\begin{array}{c}n-3 \\ n_{1}-3\end{array}\right)$ is defined to be zero if $n_{1}=2$. These formulas easily imply that

$$
\begin{gathered}
\left(n_{1}-2\right) ! n_{2} ! n_{3} ! \cdots n_{k} ! c_{3}=(n-2) ! \\
\left(n_{1}-1\right) ! n_{2} ! n_{3} ! \cdots n_{k} ! c_{4}=(n-3) !\left(\sum_{j=2}^{k} n_{j}\left(n_{j}-1\right)+\left(n_{1}-1\right)\left(n_{1}-2\right)\right) .
\end{gathered}
$$

Clearly, (4.14) is equivalent to

$$
\left(c_{3}-c_{4}\right) \operatorname{Ric}\left(e_{1}\right)+c_{4} \tau=c_{5} c .
$$

Since $M$ is not Einstein, we must have that $c_{3}=c_{4}$. Now (4.15) implies that $c_{3}-c_{4}=0$ if and only if

$$
\left(n_{2}+\cdots+n_{k}\right)\left(n_{1}-1\right)=\sum_{j=2}^{k} n_{j}\left(n_{j}-1\right) .
$$

Using the assumption $n_{1} \leq n_{j}$, this implies that $n_{1}=\ldots=n_{k}$.

So $M$ is an $S\left(n_{1}, \ldots, n_{k}\right)$-space with $n_{1}=\ldots=n_{k}$ and $n_{1}+\cdots+n_{k}=n$. Put $n_{1}=j$. Let $\left\{e_{1}, \ldots, e_{2 j}\right\}$ be an orthonormal set of vectors and let $L_{3}, \ldots, L_{k}$ be mutually orthogonal $j$-dimensional spaces, which are also orthogonal to $\left\{e_{1}, \ldots, e_{2 j}\right\}$. Then

$$
\begin{aligned}
\tau_{1 \cdots j} & +\tau_{j+1 \cdots 2 j}+\tau\left(L_{3}\right)+\cdots+\tau\left(L_{k}\right) \\
& =\tau_{1 \cdots j-1}+1+1+\tau_{j j+2 \cdots 2 j}+\tau\left(L_{3}\right)+\cdots+\tau\left(L_{k}\right),
\end{aligned}
$$

which obviously implies (3.1), so $M$ is conformally flat. 
Before going into the second case, we first prove the converse. So we suppose that $M^{n}$ is conformally flat, and suppose $n=k j$ for some natural numbers $k>1$ and $j>1$. Let $\left\{e_{1}, \ldots, e_{k j}\right\}$ be any orthonormal basis of $M$; then (3.1) easily implies that

$$
\tau_{1 \cdots j}+\tau_{j+1 \cdots 2 j}+\ldots+\tau_{(k-1) j+1 \cdots k j}
$$

does not depend on the order of the indices. Moreover, if we perform a rotation in the $\left(e_{1}, e_{2}\right)$-plane, we obviously don't change the value of (4.17). Hence, since the ordering of the indices is not important, we obtain that, if we perform a rotation in any $\left(e_{i}, e_{m}\right)$-plane, then the value of (4.17) remains the same. Since any two orthogonal bases can be mapped into each other by performing consecutive rotations in coordinate planes, we obtain that (4.17) is independent of the choice of orthonormal basis. Hence $M$ is an $S(j, \ldots, j)$-space.

Case 2. $M$ is Einstein.

Since $M$ is an $S\left(n_{1}, \ldots, n_{k}\right)$-space, we have (4.9). Now, for any permutation $\sigma$ of $\{3, \ldots, n\}$, there is a corresponding equation similar to (4.9). By summing up all such equations, we obtain

$$
\left(d_{1}-2 d_{2}+d_{3}\right) K_{12}+\left(d_{2}-d_{3}\right)\left(\operatorname{Ric}\left(e_{1}\right)+\operatorname{Ric}\left(e_{2}\right)\right)+d_{3} \tau=d_{1} c,
$$

where $d_{1}=c_{3}$, and $d_{2}, d_{3}$ are constants such that

$$
\begin{aligned}
& d_{2}=\left(\begin{array}{c}
n-3 \\
n_{1}-3
\end{array}\right)\left(\begin{array}{c}
n-n_{1} \\
n_{2}
\end{array}\right) \cdots\left(\begin{array}{c}
n-n_{1}-\cdots-n_{k-1} \\
n_{k}
\end{array}\right) \\
d_{3} & =\left(\begin{array}{c}
n-4 \\
n_{1}-4
\end{array}\right)\left(\begin{array}{c}
n-n_{1} \\
n_{2}
\end{array}\right) \cdots\left(\begin{array}{c}
n-n_{1}-\cdots-n_{k-1} \\
n_{k}
\end{array}\right) \\
& +\left(\begin{array}{c}
n-4 \\
n_{2}-2
\end{array}\right)\left(\begin{array}{c}
n-n_{2}-2 \\
n_{1}-2
\end{array}\right)\left(\begin{array}{c}
n-n_{1}-n_{2} \\
n_{3}
\end{array}\right) \cdots\left(\begin{array}{c}
n-n_{1}-\cdots-n_{k-1} \\
n_{k}
\end{array}\right) \\
& +\cdots \\
& +\left(\begin{array}{c}
n-4 \\
n_{k}-2
\end{array}\right)\left(\begin{array}{c}
n-n_{k}-2 \\
n_{1}-2
\end{array}\right)\left(\begin{array}{c}
n-n_{1}-n_{k} \\
n_{2}
\end{array}\right) \cdots\left(\begin{array}{c}
n-n_{1}-\cdots-n_{k-2}-n_{k} \\
n_{k-1}
\end{array}\right) .
\end{aligned}
$$

These formulas easily imply that

$$
\begin{gathered}
\left(n_{1}-3\right) ! n_{2} ! n_{3} ! \cdots n_{k} ! d_{2}=(n-3) ! \text { unless when } n_{1}=2, \text { then } d_{2}=0 \\
\left(n_{1}-2\right) ! n_{2} ! n_{3} ! \cdots n_{k} ! d_{3}=(n-4) !\left(\sum_{j=2}^{k} n_{j}\left(n_{j}-1\right)+\left(n_{1}-2\right)\left(n_{1}-3\right)\right) .
\end{gathered}
$$

From (4.15) and (4.19) it follows that $d_{1}-2 d_{2}+d_{3}=0$ if and only if

$$
(n-2)(n-3)+\left(n_{1}-2\right)\left(n_{1}-3\right)+\sum_{j=2}^{k} n_{j}\left(n_{j}-1\right)=2(n-3)\left(n_{1}-2\right) .
$$

Formula (4.20) is equivalent to

$$
0=\left(n-n_{1}\right)\left(n-n_{1}-1\right)+\sum_{j=2}^{k} n_{j}\left(n_{j}-1\right)
$$

which is impossible. Therefore, from (4.18) and the hypothesis that $M$ is Einstein, we obtain that $M$ is a Riemannian space form. 


\section{The IMMERSIONS Which REALIZE THE EQUALITY IN (1.3)}

In this final section, we prove the following theorem.

Theorem 5. Let $\left(n_{1}, \ldots, n_{k}\right) \in \mathcal{S}(n)$ and $M$ be a Riemannian n-manifold isometrically immersed in a Riemannian space form $R^{m}(c)$ of constant sectional curvature c. Then $M$ satisfies

$$
\hat{\delta}\left(n_{1}, \ldots, n_{k}\right)=\frac{n^{2}\left(n+k-1-\sum n_{j}\right)}{2\left(n+k-\sum n_{j}\right)} H^{2}+\frac{1}{2}\left(n(n-1)-\sum_{j=1}^{k} n_{j}\left(n_{j}-1\right)\right) c
$$

identically if and only if $M$ is a Riemannian space form and the immersion is totally umbilical.

Proof. Assume that $M$ is a submanifold in a Riemannian space form $R^{m}(c)$ which satisfies equality (5.1). Then, by (1.3) and (1.4), we have

$$
\delta\left(n_{1}, \ldots, n_{k}\right)=\hat{\delta}\left(n_{1}, \ldots, n_{k}\right),
$$

and

$$
\begin{aligned}
\tau-\left\{\tau\left(L_{1}\right)+\cdots+\tau\left(L_{k}\right)\right\} & =\frac{n^{2}\left(n+k-1-\sum n_{j}\right)}{2\left(n+k-\sum n_{j}\right)} H^{2} \\
& +\frac{1}{2}\left(n(n-1)-\sum_{j=1}^{k} n_{j}\left(n_{j}-1\right)\right) c
\end{aligned}
$$

for any mutually orthogonal $k$-plane sections $L_{1}, \ldots, L_{k} \subset T_{p} M, p \in M$, with $\operatorname{dim} L_{j}=n_{j}, j=1, \ldots, k$.

Let $\left\{e_{1}, \ldots, e_{n}, e_{n+1}, \ldots, e_{m}\right\}$ be an orthonormal basis at a point $p$ such that $L_{j}$ is spanned by $e_{n_{1}+\cdots+n_{j-1}+1}, \ldots, e_{n_{1}+\cdots+n_{j}}$, for $j=1, \ldots, k$. From (5.2) and from the proof of (1.4) given in [3], we know that the shape operators of $M$ at $p$ take the following forms:

$$
A_{r}=\left[\begin{array}{cccccc}
A_{1}^{r} & \ldots & 0 & 0 & \ldots & 0 \\
\vdots & \ddots & \vdots & \vdots & & \vdots \\
0 & \ldots & A_{k}^{r} & 0 & \ldots & 0 \\
0 & \ldots & 0 & \mu_{r} & \ldots & 0 \\
\vdots & \ddots & \vdots & \vdots & \ddots & \vdots \\
0 & \ldots & 0 & 0 & \ldots & \mu_{r}
\end{array}\right], \quad r=n+1, \ldots, m,
$$

where each $A_{j}^{r}$ is an $n_{j} \times n_{j}$ submatrix such that

$$
\operatorname{trace}\left(A_{1}^{r}\right)=\cdots=\operatorname{trace}\left(A_{k}^{r}\right)=\mu_{r}, \quad r=n+1, \ldots, m .
$$

Since $L_{1}, \ldots, L_{k}$ can be chosen to be any mutually orthogonal $k$-plane sections in $T_{p} M$, the above conditions imply that each $A_{r}$ is proportional to the identity matrix. Hence $M$ is a totally umbilical submanifold in $R^{m}(c)$.

The converse is easy to verify. 


\section{REFERENCES}

[1] M. Berger, La géométrie métrique des variétés Riemanniennes, Élie Cartan et les Mathématiques d'Aujourd'hui, Astérisque, $\mathrm{N}^{\circ}$ Hors Série, Société Mathématique de France 1985, pp. 9-66. MR 89b:53076

[2] B. Y. Chen, Some pinching and classification theorems for minimal submanifolds, Archiv der Math. 60 (1993), 568-578. MR 94d:53093

[3] B. Y. Chen, Some new obstructions to minimal and Lagrangian isometric immersions, Japan. J. Math. (to appear).

[4] B. Y. Chen, Strings of Riemannian invariants, inequalities, ideal immersions and their applications, in: Proc. 3rd Pacific Rim Geom. Conf. (International Press, Cambridge, MA) (1998) (to appear).

[5] R. S. Kulkarni, Curvature structures and conformal transformations, Bull. Amer. Math. Soc. 75 (1969), 91-94. MR 38:1628

[6] R. Osserman, Curvature in the eighties, Amer. Math. Monthly 97 (1990), 731-756. MR 91i:53001

[7] I. M. Singer and J. A. Thorpe, The curvature of 4-dimensional Einstein spaces, Global Analysis, Princeton University Press (1969), 355-365. MR 41:959

[8] L. Verstraelen and G. Zafindratafa, On the sectional curvature of conharmonically flat spaces, Rend. Sem. Mat. Messina 1 (1991), 247-254. MR 95f:53088

Department of Mathematics, Michigan State University, East Lansing, Michigan 48824

E-mail address: bychen@math.msu.edu

Departement Wiskunde, Katholieke Universiteit Leuven, Celestijnenlaan 200B, B3001 Leuven, Belgium

E-mail address: Franki.Dillen@wis.kuleuven.ac.be

E-mail address: Leopold.Verstraelen@wis.kuleuven.ac.be

E-mail address: Luc.Vrancken@wis.kuleuven.ac.be

Group of Exact Sciences, Katholieke Universiteit Brussel, Vrijheidslaan 17, B-1080 Brussel, Belgium

E-mail address: Leopold.Verstraelen@wis.kuleuven.ac.be 